\title{
A Paternò-Büchi approach to the synthesis of merrilactone A
}

\author{
Jone Iriondo-Alberdi, Jesus Perea Buceta and Michael F. Greaney \\ University of Edinburgh, School of Chemistry, Joseph Black Building, King's Buildings, \\ West Mains Road, Edinburgh, EH9 3JJ, UK \\ Michael.Greaney@ed.ac.uk
}

Supporting Information

Part A: Experimental Procedures

\section{General}

NMR spectra were recorded on a Brüker dpx360 (360 MHz) instrument and calibrated to residual solvent peaks: proton $\left(\mathrm{CDCl}_{3} 7.26 \mathrm{ppm}\right)$ and carbon $\left(\mathrm{CDCl}_{3} 77 \mathrm{ppm}\right)$. The ${ }^{1} \mathrm{H}$ data is presented as follows: chemical shift (in ppm on the $\delta$ scale), multiplicity ( $\mathrm{s}=$ singlet, $\mathrm{d}=$ doublet, $\mathrm{t}=$ =triplet, $\mathrm{q}=$ quartet, $\mathrm{m}=$ multiplet), the coupling constant $(\mathrm{J}$, in hertz) and integration. The ${ }^{13} \mathrm{C}$ data is reported as the ppm on the $\delta$ scale followed by the interpretation. IR spectra were recorded on a JASCO FT/IR-460 plus instrument using 4 $\mathrm{mm}$ sodium chloride disks. The wavelengths of the maximum absorbance $\left(v_{\max }\right)$ are quoted in $\mathrm{cm}^{-1}$. Electrospray high resolution mass spectrometry was performed by the EPSRC National Mass Spectrometry Service Centre, Swansea, using a Finnigan MAT 900 XLT double focusing mass spectrometer. FAB HRMS was carried out by the University of Edinburgh School of Chemistry mass spectrometry service using a Kratos MS50 instrument. The data is recorded as the ionisation method followed by the calculated and measured masses. TLC was performed on Merck 60F254 silica plates and visualised by UV light and/or anisaldehyde or potassium permanganate stains. The compounds were purified by wet flash chromatography using Merck Kieselgel 60 (particle size 35-70) silica under a positive pressure. The eluent is quoted as a percentage. All solvents were dried before use unless other wise stated. $\mathrm{Et}_{2} \mathrm{O}$ was dried over sodium with benzophenone as an indicator. DCM was distilled over calcium hydride. All other chemicals were purchased from a chemical supplier and used as received.

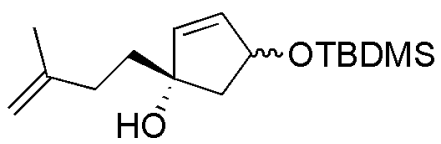

4-(tert-Butyl-dimethyl-silanyloxy)-1-(3'-methyl-but-3'-enyl)-cyclopent-2-enol (7)

To a cold $\left(-78{ }^{\circ} \mathrm{C}\right)$ solution of 4-iodo-2-methyl-but-1-ene (5.55 g, $28.30 \mathrm{mmol}, 2$ equiv) in anhydrous $\mathrm{Et}_{2} \mathrm{O}(50 \mathrm{~mL})$ under a $\mathrm{N}_{2}$ atmosphere was added dropwise ${ }^{\mathrm{t}} \mathrm{BuLi}(1.7 \mathrm{M}$ solution in pentane, $34.96 \mathrm{~mL}, 4.2$ equiv). The mixture was kept stirring for $1 \mathrm{hr}$ and was then allowed to warm up to rt and stirred for a further $1 \mathrm{hr}$. This solution was then cooled to $-78{ }^{\circ} \mathrm{C}$ and added dropwise to a solution of 4-(tert-butyl-dimethyl-silanyloxy)cyclopent-2-enone (6) (3.00 g, $14.15 \mathrm{mmol}, 1$ eq.) in anhydrous $\mathrm{Et}_{2} \mathrm{O}$ (25 mL). The 
reaction mixture was kept stirring at $-78{ }^{\circ} \mathrm{C}$ until completion $(30 \mathrm{~min})$ and it was quenched by addition of a saturated aqueous solution of $\mathrm{NH}_{4} \mathrm{Cl}(50 \mathrm{~mL})$. The aqueous layer was extracted with $\mathrm{Et}_{2} \mathrm{O}(3 \times 50 \mathrm{~mL})$ and the combined organic layers were dried over $\mathrm{MgSO}_{4}$ and the solvent evaporated to afford $3.90 \mathrm{~g}$ of a yellow oil. Purification by flash column chromatography $\left(\mathrm{SiO}_{2}, \mathrm{Hex} / \mathrm{Et}_{2} \mathrm{O}\right.$ 4:1) afforded 4-(tert-butyl-dimethylsilanyloxy)-1-(3'-methyl-but-3'-enyl)-cyclopent-2-enol (7) (3.56 g, $12.60 \mathrm{mmol}, 89 \%$ yield) as a 4/1 mixture of cis/trans diastereomers. ${ }^{1} \mathbf{H}$ NMR $\left(\mathrm{CDCl}_{3}, 360 \mathrm{MHz}\right)$ (cis diastereoisomer) $\delta 5.88(\mathrm{dd}, J=5.57,0.71 \mathrm{~Hz}, 1 \mathrm{H}), 5.86(\mathrm{dd}, J=5.57,2.00 \mathrm{~Hz}, 1 \mathrm{H}), 4.64-$ $4.71(\mathrm{~m}, 3 \mathrm{H}), 2.36(\mathrm{dd}, J=13.75,6.64 \mathrm{~Hz}, 1 \mathrm{H}), 2.00-2.09(\mathrm{~m}, 2 \mathrm{H}), 1.68-1.75(\mathrm{~m}, 9 \mathrm{H})$, 0.85-0.91 (m, 9H), $0.08(\mathrm{~s}, 6 \mathrm{H}) ;{ }^{13} \mathbf{C ~} \mathbf{N M R}\left(\mathrm{CDCl}_{3}, 90 \mathrm{MHz}\right)$ (cis diastereoisomer) $\delta$ 145.91 (q), $138.87(\mathrm{CH}), 135.49(\mathrm{CH}), 109.60\left(\mathrm{CH}_{2}\right), 83.62(\mathrm{q}), 75.31(\mathrm{CH}), 49.02$ $\left(\mathrm{CH}_{2}\right), 37.92\left(\mathrm{CH}_{2}\right), 32.44\left(\mathrm{CH}_{2}\right), 25.86\left(\mathrm{CH}_{3}\right), 22.69(\mathrm{q}), 18.12\left(\mathrm{CH}_{3}\right),-4.66\left(\mathrm{CH}_{3}\right)$; IR (thin film) $v$ 3408, 2954, 2930, 2857, 1082, 901, 836, 775, 667; HRMS (ES) $m / z$ calcd for $\mathrm{C}_{16} \mathrm{H}_{34} \mathrm{O}_{2} \mathrm{NSi}\left[\mathrm{M}+\mathrm{NH}_{4}\right]^{+}$300.2353. Found 300.2353.

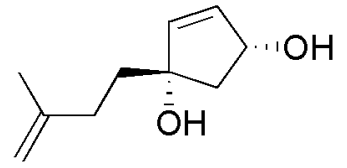

\section{1-(3-Methyl-but-3-enyl)-cyclopent-4-ene-1,3-diol}

To a solution of cis-4-(tert-butyl-dimethyl-silanyloxy)-1-(3-methyl-but-3-enyl)cyclopent-2-enol (7) (1.54 g, $5.74 \mathrm{mmol}, 1$ equiv) in THF (40 mL) at rt was added TBAF (1.0 M solution in THF, $11.47 \mathrm{~mL}, 11.47 \mathrm{mmol}, 2$ equiv). The reaction mixture was stirred overnight and upon completion it was quenched by the addition of a saturated aqueous solution of $\mathrm{NH}_{4} \mathrm{Cl}(40 \mathrm{~mL})$. The aqueous layer was extracted with $\mathrm{Et}_{2} \mathrm{O}(3 \times 40$ $\mathrm{mL}$ ) and the combined organic layers were dried over $\mathrm{Na}_{2} \mathrm{SO}_{4}$ and solvent evaporated to afford $1.75 \mathrm{~g}$ of a brown oil. Purification of the residue by column chromatography $\left(\mathrm{SiO}_{2}\right.$, EtOAc $\left.100 \%\right)$ afforded 1-(3-methyl-but-3-enyl)-cyclopent-4-ene-1,3-diol $(0.85 \mathrm{~g}$, $5.05 \mathrm{mmol}, 88 \%$ yield) as a yellow oil. ${ }^{1} \mathbf{H} \mathbf{~ N M R}\left(\mathrm{CDCl}_{3}, 360 \mathrm{MHz}\right) \delta 5.82-5.90(\mathrm{~m}, 2 \mathrm{H})$, 4.57-4.60 (m, 3H), 2.40 (dd, $J=14.58,7.25 \mathrm{~Hz}, 1 \mathrm{H}), 1.94-2.03(\mathrm{~m}, 2 \mathrm{H}), 1.65-1.74(\mathrm{~m}$, $6 \mathrm{H}) ;{ }^{13} \mathrm{C} \mathrm{NMR}\left(\mathrm{CDCl}_{3}, 90 \mathrm{MHz}\right) \delta 145.56(\mathrm{q}), 139.51(\mathrm{CH}), 134.73(\mathrm{CH}), 109.68\left(\mathrm{CH}_{2}\right)$, 83.80 (q), $74.92(\mathrm{CH}), 47.53\left(\mathrm{CH}_{2}\right), 38.35\left(\mathrm{CH}_{2}\right), 32.31\left(\mathrm{CH}_{2}\right), 22.64\left(\mathrm{CH}_{3}\right)$; IR (thin film) $v$ 3334, 3073, 2966, 2937, 2857, 1649, 1445, 1096, 885; HRMS (ES) $\mathrm{m} / \mathrm{z}$ calcd for $\mathrm{C}_{10} \mathrm{H}_{20} \mathrm{O}_{2} \mathrm{~N}\left[\mathrm{M}+\mathrm{NH}_{4}\right]^{+}$186.1489. Found 186.1490.

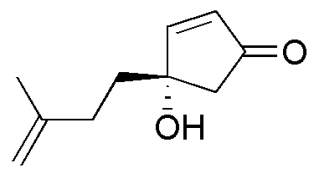

\section{4-Hydroxy-4-(3-methyl-but-3-enyl)-cyclopent-2-enone (8)}

To a solution of 1-(3-methyl-but-3-enyl)-cyclopent-4-ene-1,3-diol (0.65 g, $3.57 \mathrm{mmol}, 1$ equiv) in dry DCM $(15 \mathrm{~mL})$ was added PDC (2.05 g, $5.35 \mathrm{mmol}, 1.5$ equiv) at $0{ }^{\circ} \mathrm{C}$. The reaction was stirred overnight at $\mathrm{rt}$. The reaction mixture was then filtered through celite with thorough DCM washing. The organic solvent was evaporated and the residue was 
purified by column chromatography ( $\mathrm{SiO}_{2}$, EtOAc 100\%) to afford 4-hydroxy-4-(3methyl-but-3-enyl)-cyclopent-2-enone (8) $(0.41 \mathrm{~g}, 2.50 \mathrm{mmol}, 70 \%$ yield $)$ as a yellow oil. ${ }^{1} \mathbf{H}$ NMR $\left(\mathrm{CDCl}_{3}, 360 \mathrm{MHz}\right) \delta 7.44(\mathrm{~d}, J=5.67 \mathrm{~Hz}, 1 \mathrm{H}), 6.14(\mathrm{~d}, J=5.67 \mathrm{~Hz}, 1 \mathrm{H}), 4.76$ $(\mathrm{s}, 1 \mathrm{H}), 4.73(\mathrm{~s}, 1 \mathrm{H}), 2.52(\mathrm{ABq}, J=44.35,18.44 \mathrm{~Hz}, 2 \mathrm{H}), 2.03-2.20(\mathrm{~m}, 2 \mathrm{H}), 1.82-1.98$ $(\mathrm{m}, 2 \mathrm{H}), 1.75(\mathrm{~s}, 3 \mathrm{H}) ;{ }^{13} \mathrm{C} \mathrm{NMR}\left(\mathrm{CDCl}_{3}, 90 \mathrm{MHz}\right) \delta 206.60(\mathrm{q}), 165.41(\mathrm{CH}), 144.94(\mathrm{q})$, $133.53(\mathrm{CH}), 110.69\left(\mathrm{CH}_{2}\right), 79.24(\mathrm{q}), 48.88\left(\mathrm{CH}_{2}\right), 38.04\left(\mathrm{CH}_{2}\right), 32.32\left(\mathrm{CH}_{2}\right), 22.53$ $\left(\mathrm{CH}_{3}\right)$; IR (thin film) v 3398, 2933, 1717, 1676, 1648, 1590, 1406, 1124, 889, 804; HRMS (ES) $m / z$ calcd for $\mathrm{C}_{10} \mathrm{H}_{18} \mathrm{O}_{2} \mathrm{~N}\left[\mathrm{M}+\mathrm{NH}_{4}\right]^{+}$184.1332. Found 184.1334.

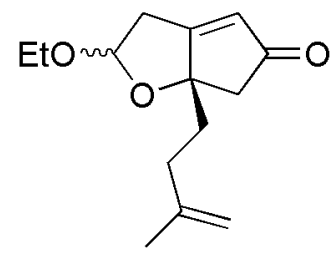

2-Ethoxy-6a-(3-methylbut-3-enyl)-2,3,6,6a-tetrahydrocyclopenta[b]furan-5-one (11) To a solution of 4-hydroxy-4-(3-methyl-but-3-enyl)-cyclopent-2-enone (8) (0.12 g, 0.72 mmol, 1 equiv) in ethyl vinyl ether $\left(2.76 \mathrm{~mL}, 28.87 \mathrm{mmol}, 40\right.$ equiv), $\mathrm{Pd}(\mathrm{OAc})_{2}(0.16 \mathrm{~g}$, $0.72 \mathrm{mmol}, 1$ equiv) was added and the resulting mixture was stirred at $\mathrm{rt}$ for $3 \mathrm{hr}$. The mixture was then filtered through celite with thorough washing with DCM. Evaporation of the solvent afforded a residual orange oil. Purification by column chromatography $\left(\mathrm{SiO}_{2}\right.$ saturated with $\left.\mathrm{Et}_{3} \mathrm{~N}, \mathrm{Et}_{2} \mathrm{O} / \mathrm{Hex} 4: 6\right)$ afforded 2-ethoxy-6a-(3-methylbut-3-enyl)2,3,6,6a-tetrahydrocyclopenta[b]furan-5-one (11) (0.11 g, $0.47 \mathrm{mmol}$, 65\%yield, 1:1 mixture of diastereomeric acetals) as a yellow oil. ${ }^{1} \mathbf{H} \mathbf{~ N M R}\left(\mathrm{CDCl}_{3}, 360 \mathrm{MHz}\right) \delta 6.05(\mathrm{~d}$, $J=2.38 \mathrm{~Hz}, 1 \mathrm{H}), 5.91(\mathrm{~m}, 1 \mathrm{H}), 5.42(\mathrm{~d}, J=6.05 \mathrm{~Hz}, 1 \mathrm{H}), 5.34(\mathrm{dd}, J=5.66,3.57 \mathrm{~Hz}$, $1 \mathrm{H}), 4.65-4.72(\mathrm{~m}, 4 \mathrm{H}), 3.91(\mathrm{~m}, 1 \mathrm{H}), 3.74(\mathrm{~m}, 1 \mathrm{H}), 3.44-3.58(\mathrm{~m}, 2 \mathrm{H}), 2.43-3.17(\mathrm{~m}$, $8 \mathrm{H}), 1.74-2.22(\mathrm{~m}, 6 \mathrm{H}), 1.73(\mathrm{~s}, 3 \mathrm{H}), 1.71(\mathrm{~s}, 3 \mathrm{H}), 1.51-1.66(\mathrm{~m}, 2 \mathrm{H}), 1.24(\mathrm{dt}, J=7.08$, $0.56 \mathrm{~Hz}, 3 \mathrm{H}), 1.17(\mathrm{dt}, J=7.08,0.59 \mathrm{~Hz}, 3 \mathrm{H}) ;{ }^{13} \mathbf{C ~ N M R}\left(\mathrm{CDCl}_{3}, 90 \mathrm{MHz}\right) \delta 205.60(\mathrm{q})$, 205.46 (q), 180.88 (q), 179.89 (q), 144.91 (q), 144.71 (q), $126.56(\mathrm{CH}), 124.96(\mathrm{CH})$, $110.26\left(\mathrm{CH}_{2}\right), 110.09\left(\mathrm{CH}_{2}\right), 105.27(\mathrm{CH}), 103.47(\mathrm{CH}), 89.55$ (q), 88.98 (q), 64.40 $\left(\mathrm{CH}_{2}\right), 63.04\left(\mathrm{CH}_{2}\right), 51.95\left(\mathrm{CH}_{2}\right), 50.08\left(\mathrm{CH}_{2}\right), 37.93\left(\mathrm{CH}_{2}\right), 36.97\left(\mathrm{CH}_{2}\right), 35.19\left(\mathrm{CH}_{2}\right)$, $34.49\left(\mathrm{CH}_{2}\right), 32.83\left(\mathrm{CH}_{2}\right), 32.50\left(\mathrm{CH}_{2}\right), 22.69\left(\mathrm{CH}_{3}\right), 22.53\left(\mathrm{CH}_{3}\right), 15.10\left(\mathrm{CH}_{3}\right), 15.07$ $\left(\mathrm{CH}_{3}\right)$; IR (thin film) $v$ 3075, 2976, 2931, 1719, 1650, 1088, 956; HRMS (FAB) $\mathrm{m} / \mathrm{z}$ calcd for $\mathrm{C}_{14} \mathrm{H}_{21} \mathrm{O}_{3}[\mathrm{MH}]^{+}$237.14907. Found 237.14902.

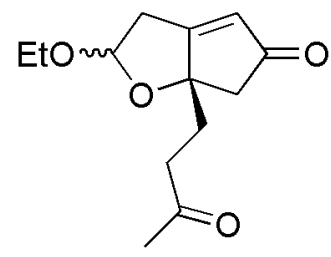

\section{2-Ethoxy-6a-(3-oxobutyl)-2,3,6,6a-tetrahydrocyclopenta[b]furan-5-one (16)}

To a solution of 2-ethoxy-6a-(3-methylbut-3-enyl)-2,3,6,6atetrahydrocyclopenta[b]furan-5-one (11) $(0.09 \mathrm{~g}, 0.38 \mathrm{mmol}, 1$ equiv) in dioxane-water 
(3:1, $16 \mathrm{~mL})$ were added 2,6-lutidine $\left(0.01 \mathrm{~mL}, 0.76 \mathrm{mmol}, 2\right.$ equiv), $\mathrm{OsO}_{4}(4 \%$ in water, $0.05 \mathrm{~mL}, 7.6 \mu \mathrm{mol}, 0.02$ equiv) and $\mathrm{NaIO}_{4}(0.33 \mathrm{~g}, 1.52 \mathrm{mmol}, 4$ equiv). The reaction was stirred at ambient temperature and monitored by TLC. After the reaction was complete, water $(10 \mathrm{~mL})$ and DCM $(10 \mathrm{~mL})$ were added. The organic layer was separated, and the aqueous layer was extracted with DCM (15 mL) 3 times. The combined organic layer was washed with brine, dried over $\mathrm{MgSO}_{4}$ and solvent evaporated to afford $0.12 \mathrm{~g}$ of a brown oil. Purification of the residue by column chromatography $\left(\mathrm{SiO}_{2}, \mathrm{Et}_{2} \mathrm{O} / \mathrm{Hex} \quad 7: 3\right)$ yielded 2-ethoxy-6a-(3-oxobutyl)-2,3,6,6atetrahydrocyclopenta[b]furan-5-one (16) $(0.04 \mathrm{~g}, 0.18 \mathrm{mmol}, 47 \%$ yield, $1: 1$ mixture of acetal diastereoisomers) as a brown oil. ${ }^{1} \mathbf{H} \mathbf{~ N M R ~}\left(\mathrm{CDCl}_{3}, 360 \mathrm{MHz}\right) \delta 6.01(\mathrm{~d}, J=2.31$ $\mathrm{Hz}, 1 \mathrm{H}), 5.90(\mathrm{~d}, J=2.36,0.88 \mathrm{~Hz}, 1 \mathrm{H}), 5.34(\mathrm{~d}, J=5.64 \mathrm{~Hz}, 1 \mathrm{H}), 5.32$ (dd, $J=3.72,5.64$ $\mathrm{Hz}, 1 \mathrm{H}), 3.85(\mathrm{~m}, 1 \mathrm{H}), 3.70(\mathrm{~m}, 1 \mathrm{H}), 3.54(\mathrm{~m}, 1 \mathrm{H}), 3.43(\mathrm{~m}, 1 \mathrm{H}), 3.08-3.17(\mathrm{~m}, 2 \mathrm{H}), 2.92$ $(\mathrm{m}, 1 \mathrm{H}), 2.76(\mathrm{ABd}, J=15.70 \mathrm{~Hz}, 1 \mathrm{H}), 2.32-2.66(\mathrm{~m}, 8 \mathrm{H}), 2.21(\mathrm{~m}, 1 \mathrm{H}), 2.15(\mathrm{~s}, 6 \mathrm{H})$, $2.04(\mathrm{~m}, 1 \mathrm{H}), 1.85(\mathrm{~m}, 1 \mathrm{H}), 1.68(\mathrm{~m}, 1 \mathrm{H}), 1.22(\mathrm{t}, J=7.09 \mathrm{~Hz}, 3 \mathrm{H}), 1.15(\mathrm{t}, J=7.08 \mathrm{~Hz}$, $3 \mathrm{H}) ;{ }^{13} \mathbf{C ~ N M R}\left(\mathrm{CDCl}_{3}, 90 \mathrm{MHz}\right) \delta 207.45(\mathrm{q}), 207.36(\mathrm{q}), 205.17$ (q), $205.10(\mathrm{q}), 180.54$ (q), $180.03(\mathrm{q}), 126.40(\mathrm{CH}), 125.16(\mathrm{CH}), 105.41(\mathrm{CH}), 103.63(\mathrm{CH}), 88.82(\mathrm{q}), 88.26$ (q), $64.51\left(\mathrm{CH}_{2}\right), 63.17\left(\mathrm{CH}_{2}\right), 51.53\left(\mathrm{CH}_{2}\right), 50.00\left(\mathrm{CH}_{2}\right), 38.83\left(\mathrm{CH}_{2}\right), 38.49\left(\mathrm{CH}_{2}\right)$, $34.89\left(\mathrm{CH}_{2}\right), 34.42\left(\mathrm{CH}_{2}\right), 32.97\left(\mathrm{CH}_{2}\right), 32.25\left(\mathrm{CH}_{2}\right), 30.22\left(\mathrm{CH}_{3}\right), 30.02\left(\mathrm{CH}_{3}\right), 15.10$ $\left(\mathrm{CH}_{3}\right), 15.07\left(\mathrm{CH}_{3}\right)$; IR (thin film) v 2976, 2929, 1716, 1650, 1089, 954; HRMS (FAB) $\mathrm{m} / \mathrm{z}$ calcd for $\mathrm{C}_{13} \mathrm{H}_{19} \mathrm{O}_{4}[\mathrm{MH}]^{+} 239.12833$. Found 239.12836 .

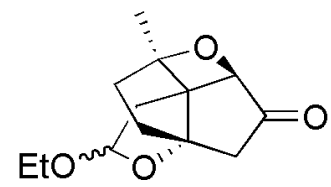

\section{Oxetane (17)}

2-Ethoxy-6a-(3-oxobutyl)-2,3,6,6a-tetrahydrocyclopenta[b]furan-5-one (16) (0.13 g, 0.54 mmol, 1 equiv) was dissolved in acetonitrile $(200 \mathrm{~mL})$ and the solution deoxygenated using a flow of nitrogen for $1 \mathrm{hr}$. The solution was then immersed in an ice bath and irradiated with a $400 \mathrm{~W}$ medium pressure mercury lamp using a pyrex filter. The reaction was monitored by TLC and after $3 \mathrm{hr}$ was stopped and the solvent evaporated. Purification of the residue by column chromatography afforded oxetane (17) $(0.12 \mathrm{~g}, 0.50$ mmol, 93\% yield, 1:1 mixture of acetal diastereoisomers) as a yellow oil and 2-ethoxy6a-(3-oxobutyl)-2,3,6,6a-tetrahydrocyclopenta[b]furan-5-one (16) (0.01 g, $0.04 \mathrm{mmol}$, $7 \%$ recovered starting material) as a yellow oil. ${ }^{1} \mathbf{H} \mathbf{~ N M R}\left(\mathrm{CDCl}_{3}, 360 \mathrm{MHz}\right) \delta 5.29(\mathrm{dd}$, $J=4.19,1.27 \mathrm{~Hz}, 1 \mathrm{H}), 5.19(\mathrm{~d}, J=4.69 \mathrm{~Hz}, 1 \mathrm{H}), 4.66$ (s, 1H), 4.60 (s, 1H), 3.71-3.79 (m, 2H), 3.37-3.47 (m, 2H), 2.86-3.04 (m, 3H), 2.60 (ABd, J=18.26 Hz, 1H), 1.87-2.32 (m, $12 \mathrm{H}), 1.63(\mathrm{~s}, 3 \mathrm{H}), 1.60(\mathrm{~s}, 3 \mathrm{H}), 1.21(\mathrm{t}, J=7.08 \mathrm{~Hz}, 3 \mathrm{H}), 1.15(\mathrm{t}, J=7.09 \mathrm{~Hz}, 3 \mathrm{H}) ;{ }^{13} \mathrm{C}$ NMR $\left(\mathrm{CDCl}_{3}, 90 \mathrm{MHz}\right) \delta 210.97(\mathrm{q}), 210.49(\mathrm{q}), 107.70(\mathrm{CH}), 105.51(\mathrm{CH}), 98.94(\mathrm{q})$, 98.84 (q), 98.72 (q), 96.65 (q), $84.43(\mathrm{CH}), 81.44(\mathrm{CH}), 62.58\left(\mathrm{CH}_{2}\right), 62.35\left(\mathrm{CH}_{2}\right), 54.57$ $\left(\mathrm{CH}_{2}\right), 51.86\left(\mathrm{CH}_{2}\right), 39.95\left(\mathrm{CH}_{2}\right), 39.20\left(\mathrm{CH}_{2}\right), 38.68\left(\mathrm{CH}_{2}\right), 38.37\left(\mathrm{CH}_{2}\right), 34.39\left(\mathrm{CH}_{2}\right)$, $34.08\left(\mathrm{CH}_{2}\right), 30.22(\mathrm{q}), 29.61(\mathrm{q}), 21.51\left(\mathrm{CH}_{3}\right), 21.18\left(\mathrm{CH}_{3}\right), 14.88\left(\mathrm{CH}_{3}\right), 14.69\left(\mathrm{CH}_{3}\right)$. IR (thin film) $v$ 2969, 2928, 1752, 1092, 1033; HRMS (ES) $\mathrm{m} / z$ calcd for $\mathrm{C}_{13} \mathrm{H}_{22} \mathrm{O}_{4} \mathrm{~N}$ $\left[\mathrm{M}+\mathrm{NH}_{4}\right]^{+}$256.1543. Found 256.1540. 
Part B: Spectroscopic Data 

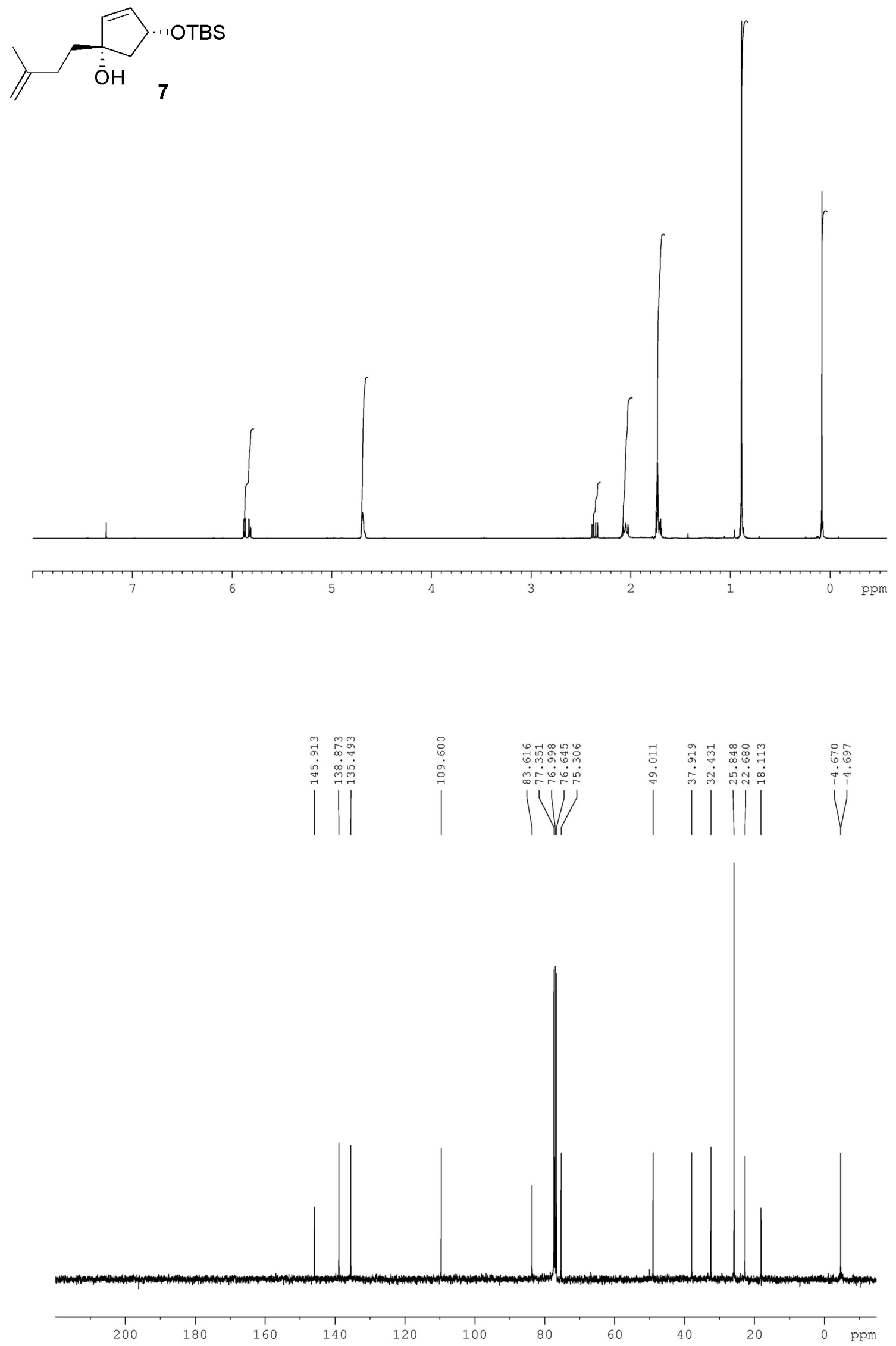
$\geqslant \overbrace{\overline{\mathrm{O}} \mathrm{H}} \cdot \mathrm{OH}$

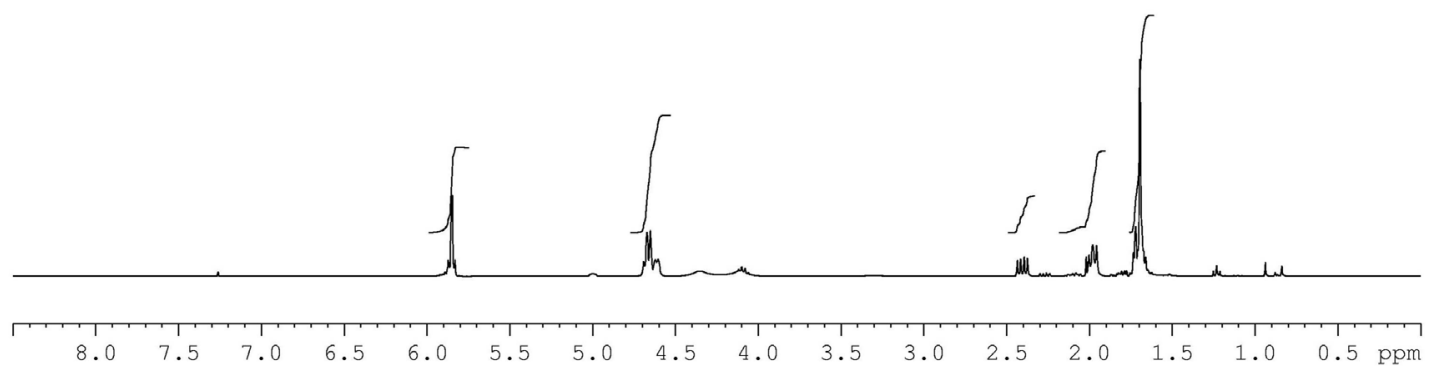

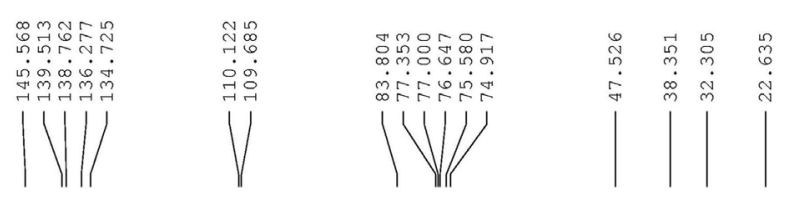

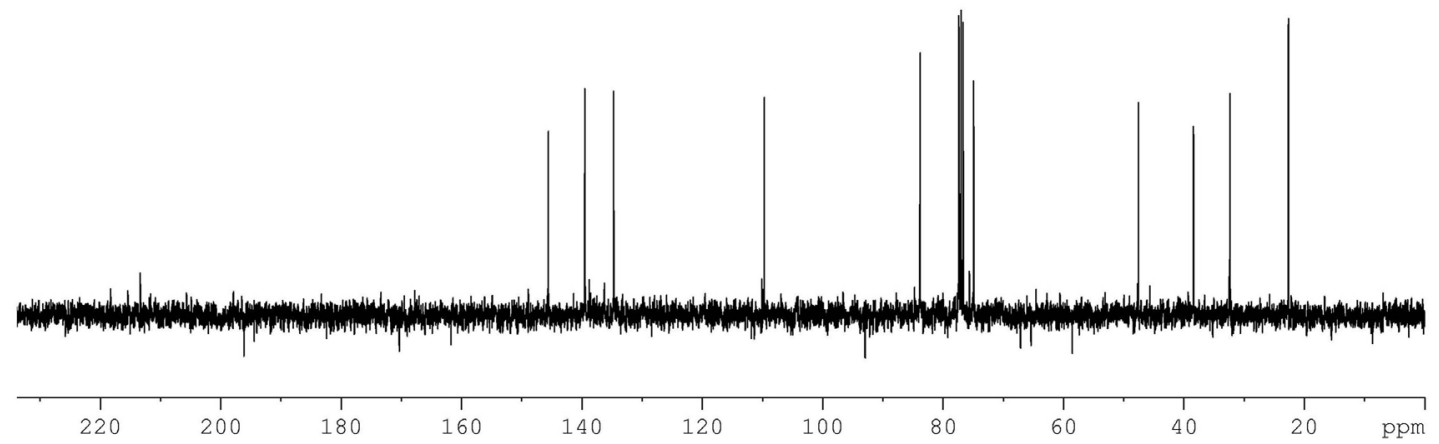



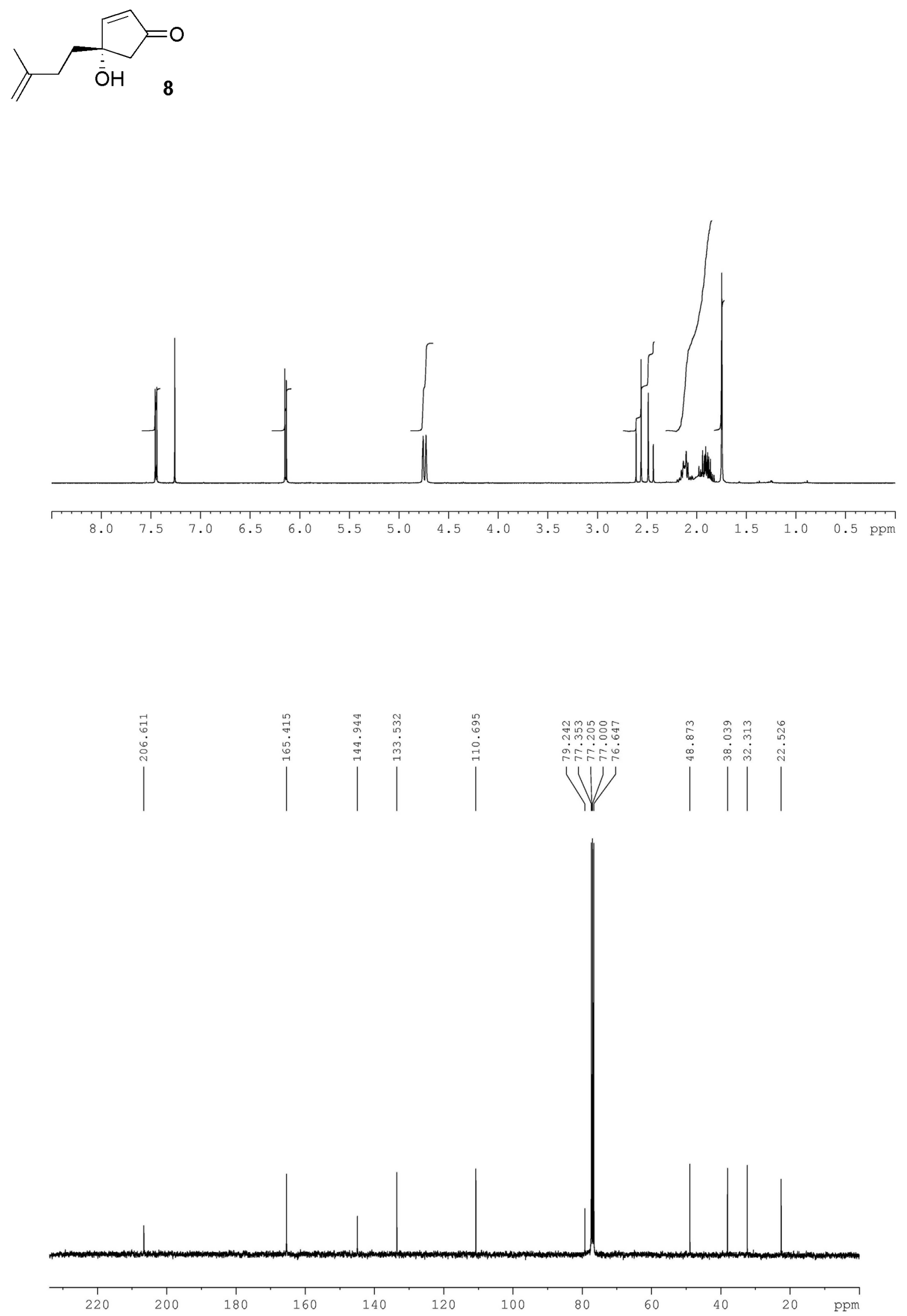

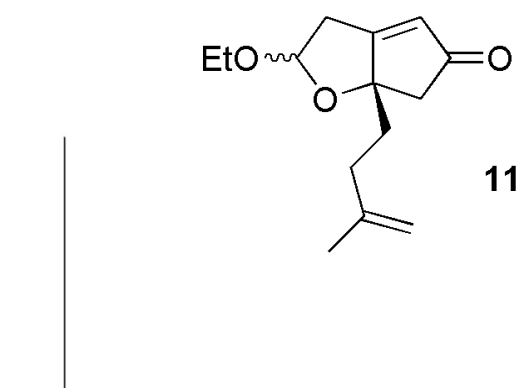

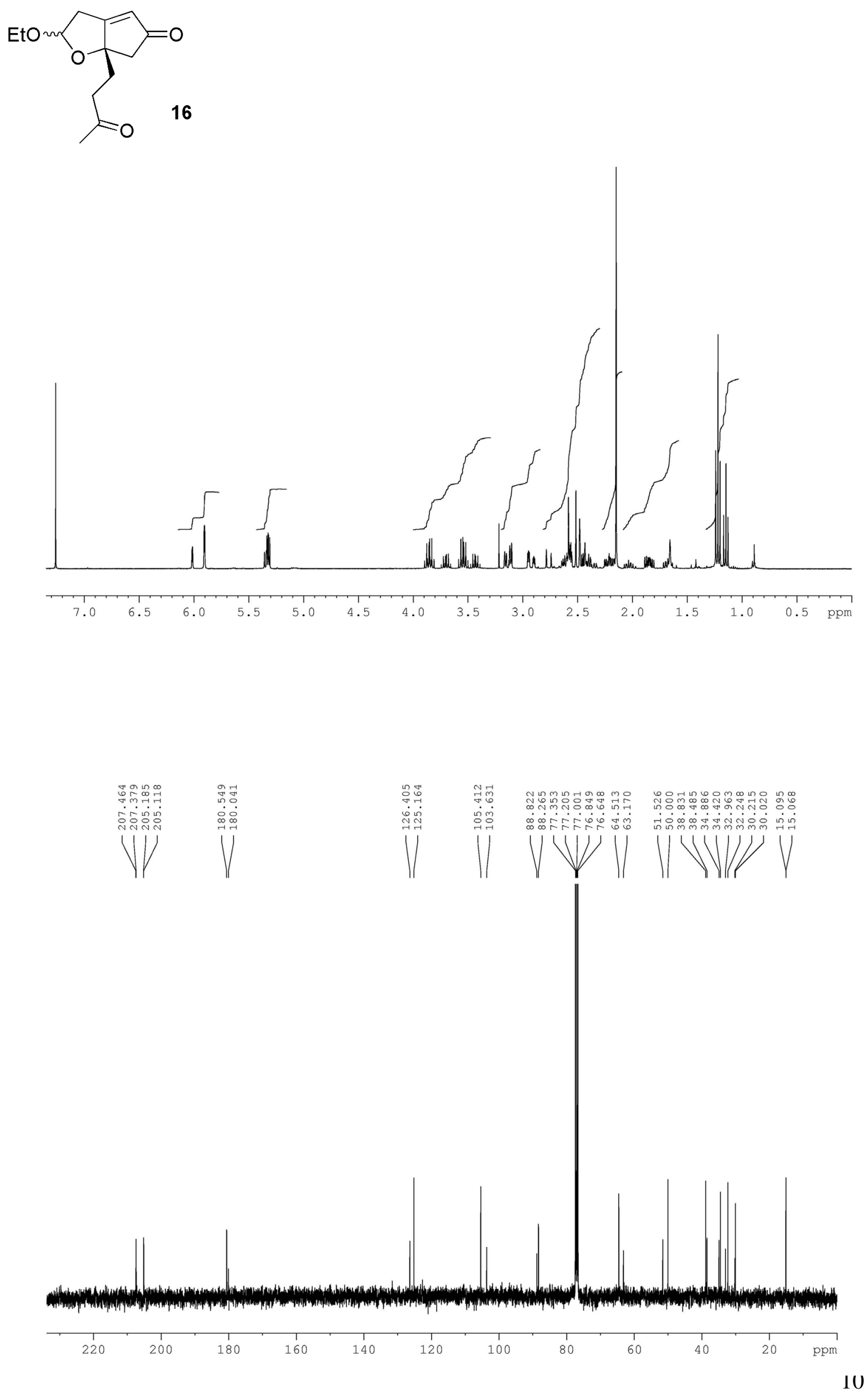
<smiles>CCO[C@@H]1O[C@@H]2CC(=O)C3O[C@@]4(C)CC1C32C4</smiles>
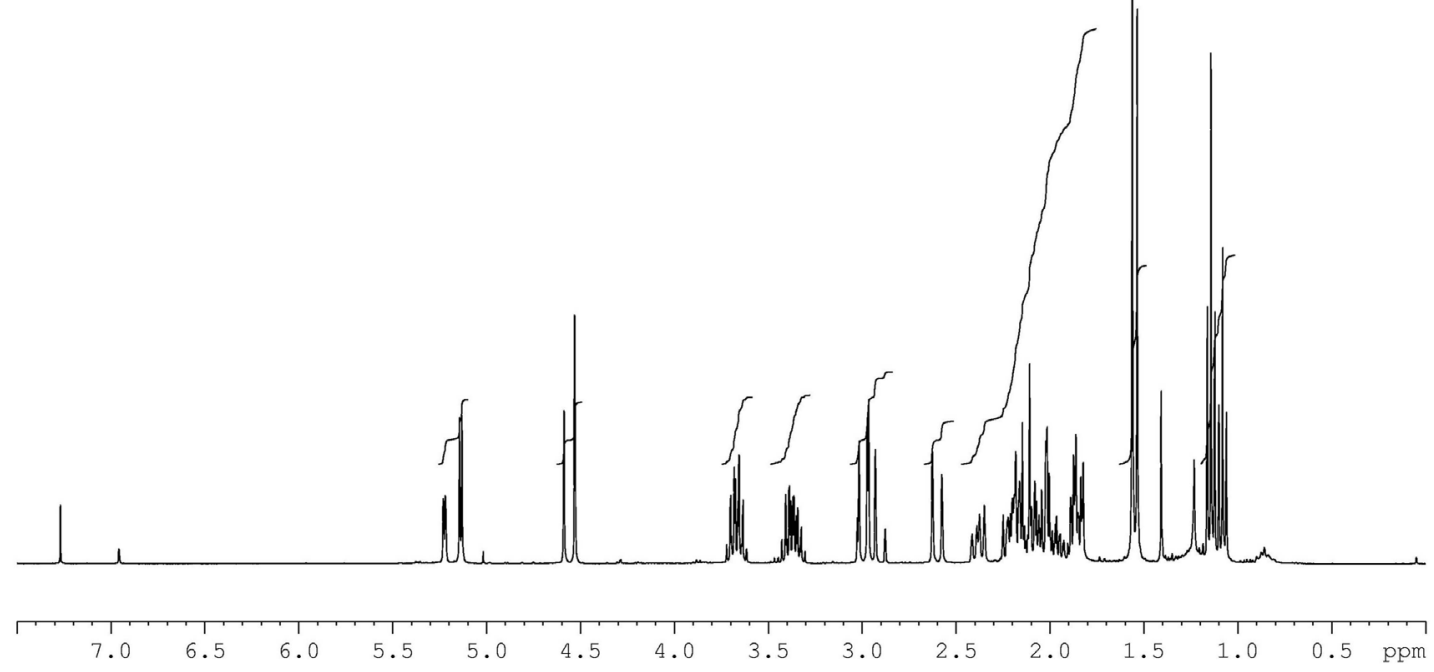
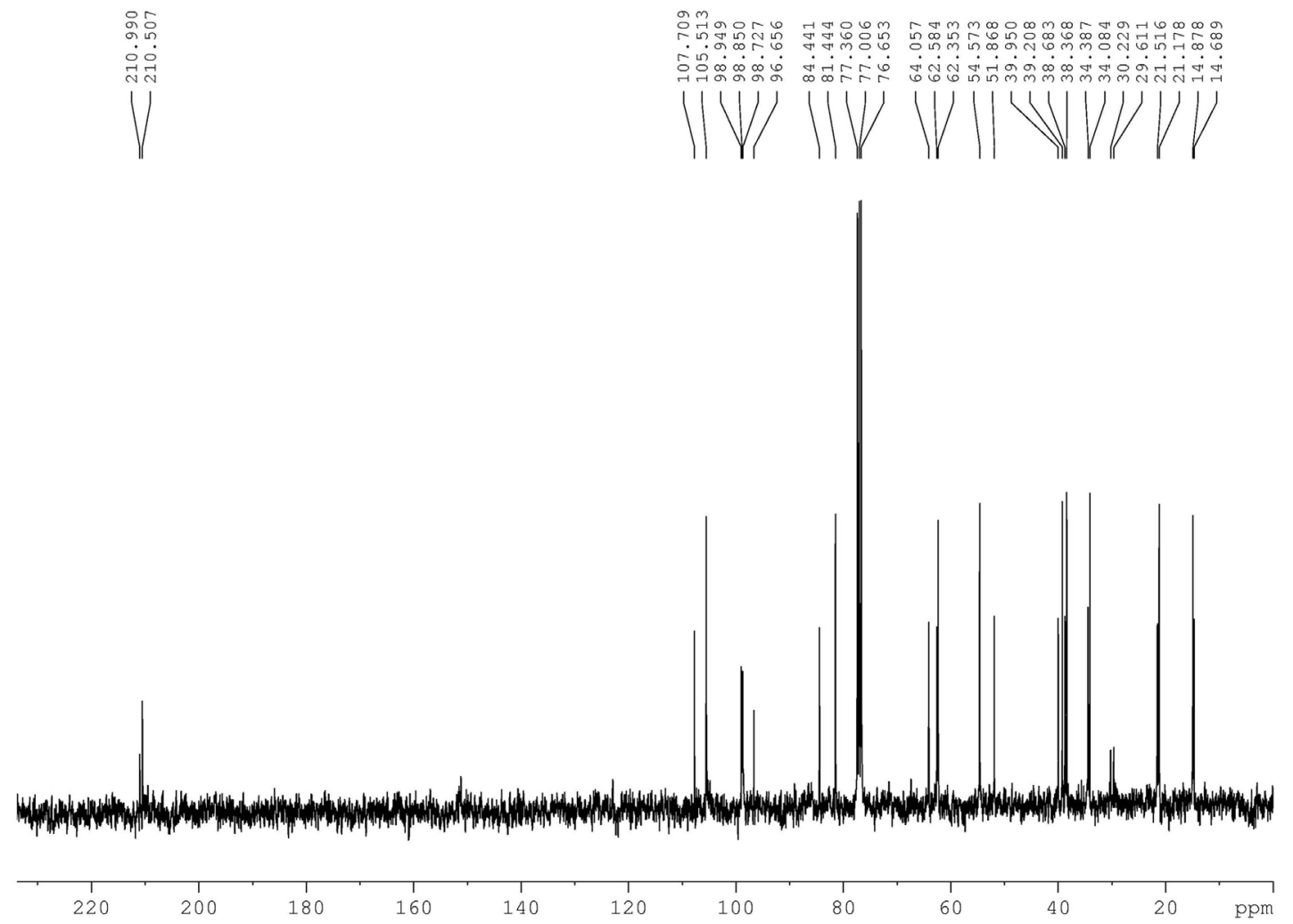


\section{COSY}

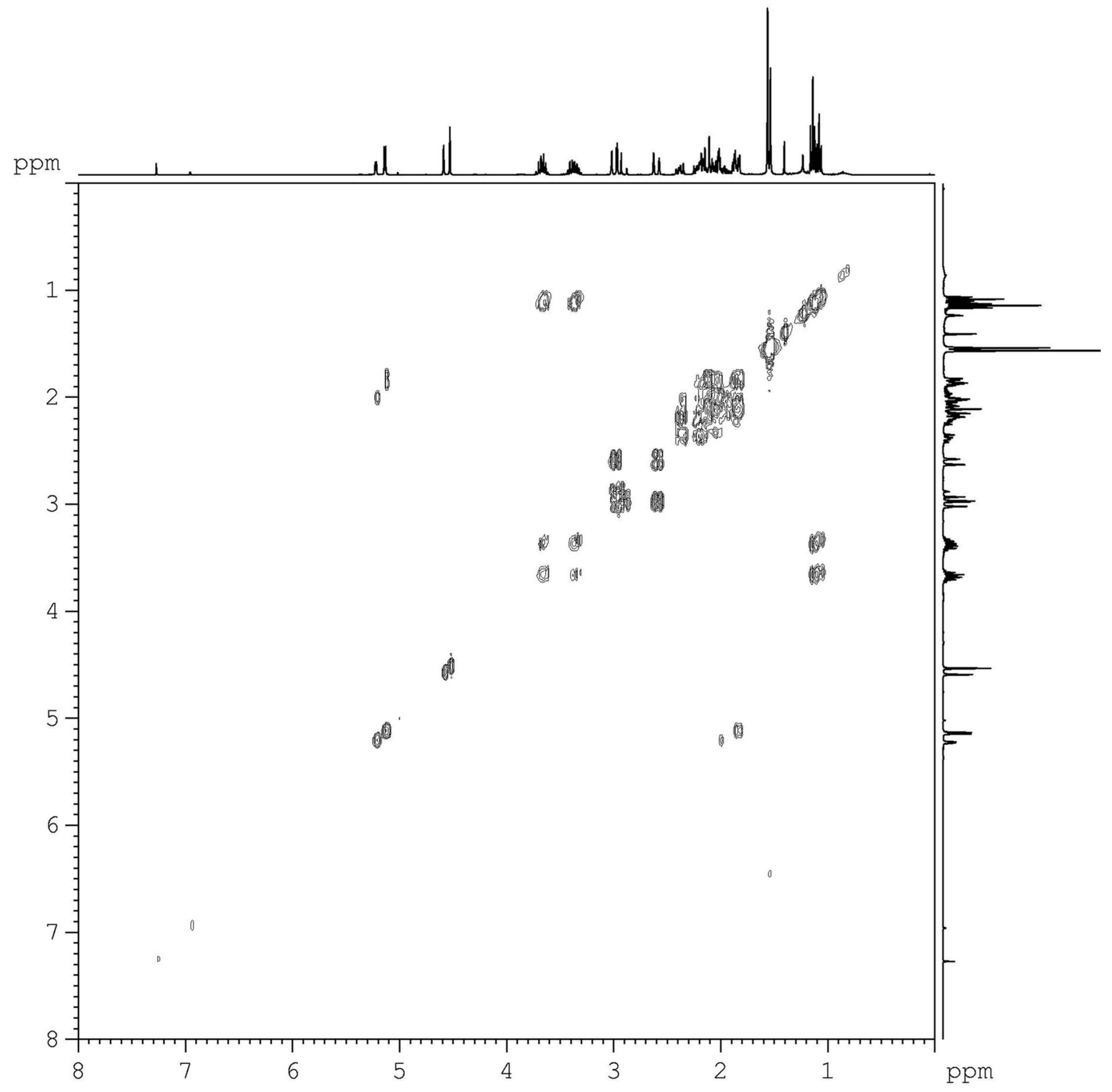


HSQC

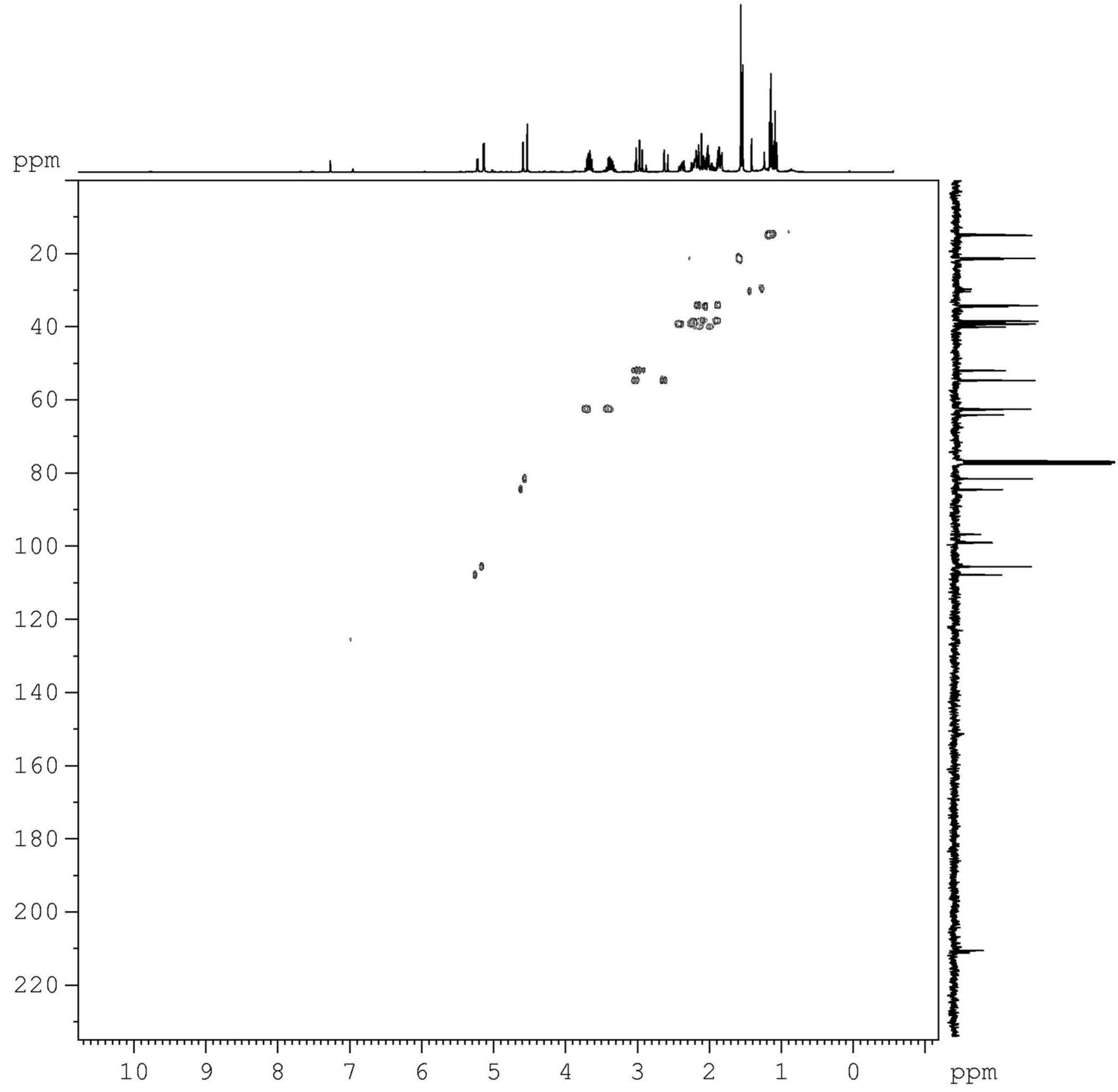

